\title{
Using induced pluripotent stem cells to investigate human neuronal phenotypes in 1q21.1 deletion and duplication syndrome
}

\author{
Gareth Chapman ${ }^{1,2} \cdot$ Mouhamed Alsaqati $^{1,3} \cdot$ Sharna Lunn ${ }^{1,2} \cdot$ Tanya Singh $^{1,2} \cdot$ Stefanie C. Linden ${ }^{1,3,4}$. \\ David E. J. Linden $\mathbb{1}^{1,3,5} \cdot$ Marianne B. M. van den Bree $\mathbb{D}^{1,3} \cdot$ Mike Ziller $^{6} \cdot$ Michael J. Owen $\mathbb{D}^{1,3} \cdot$ Jeremy Hall $\mathbb{D}^{1,3}$. \\ Adrian J. Harwood ${ }^{1,2} \cdot$ Yasir Ahmed Syed $\mathbb{D}^{1,2}$
}

Received: 16 February 2021 / Revised: 17 May 2021 / Accepted: 27 May 2021

(c) The Author(s) 2021. This article is published with open access

\begin{abstract}
Copy Number Variation (CNV) at the 1q21.1 locus is associated with a range of neurodevelopmental and psychiatric disorders in humans, including abnormalities in head size and motor deficits. Yet, the functional consequences of these CNVs (both deletion and duplication) on neuronal development remain unknown. To determine the impact of CNV at the 1q21.1 locus on neuronal development, we generated induced pluripotent stem cells from individuals harbouring 1q21.1 deletion or duplication and differentiated them into functional cortical neurons. We show that neurons with 1q21.1 deletion or duplication display reciprocal phenotype with respect to proliferation, differentiation potential, neuronal maturation, synaptic density and functional activity. Deletion of the 1q21.1 locus was also associated with an increased expression of lower cortical layer markers. This difference was conserved in the mouse model of 1q21.1 deletion, which displayed altered corticogenesis. Importantly, we show that neurons with 1q21.1 deletion and duplication are associated with differential expression of calcium channels and demonstrate that physiological deficits in neurons with 1q21.1 deletion or duplication can be pharmacologically modulated by targeting $\mathrm{Ca}^{2+}$ channel activity. These findings provide biological insight into the neuropathological mechanism underlying 1q21.1 associated brain disorder and indicate a potential target for therapeutic interventions.
\end{abstract}

Supplementary information The online version contains supplementary material available at https://doi.org/10.1038/s41380021-01182-2.

Yasir Ahmed Syed

syedy@ cardiff.ac.uk

1 Neuroscience and Mental Health Research Institute, Hadyn Ellis Building, Cardiff University, Cardiff, United Kingdom

2 School of Biosciences, The Sir Martin Evans Building, Museum Ave, Cardiff University, Cardiff, United Kingdom

3 Division of Psychological Medicine and Clinical Neurosciences (DPMCN), School of Medicine, Cardiff University, Cardiff, United Kingdom

4 Care and Public Health Research Institute, Faculty of Health, Medicine and Life Sciences, Maastricht University, Maastricht, The Netherlands

5 School of Mental Health and Neuroscience, Faculty of Health, Medicine and Life Sciences, Maastricht University, Maastricht, The Netherlands

6 MaxPlanck Institute for Psychiatry, Munich, Germany

\section{Introduction}

Investigating the biology of rare but relatively penetrant copy number variants (CNVs), provides an opportunity to understand the genetic basis of an increased susceptibility to a range of neurodevelopmental and neuropsychiatric disorders such as schizophrenia, autism, mental retardation and epilepsy [1-7]. There are now several prominent examples of pathogenic CNVs such as 1q21.1 deletions and duplications, 3q29 microduplications, 15q13.3 deletions, $16 \mathrm{p} 11.2$ deletions and duplications and 22q11.2 deletions all of which are associated with increased risk for neurodevelopmental and neuropsychiatric disorders [8-10]. These CNVs are variable in size and can be either de novo or familial [11, 12]. Furthermore, a recent study showed that the brain is the tissue which is most intolerant to CNV associated changes in gene dosage [13]. Therefore, studying the impact of these CNVs on brain development provides a window of opportunity to understand the cellular mechanisms underlying increased risk for psychiatric disorders. 
The 1q21.1 chromosomal locus (chr1: 146.57-147.39; GRCh37/hg19) contains at least four low copy repeats which render this region susceptible to non-allelic homologous recombination leading to recurrent deletions and duplications [14-16]. Although its prevalence worldwide is not clear, data from UK Biobank has provided estimates of a population frequency of $0.027 \%$ for the $1 \mathrm{q} 21.1$ deletion and $0.044 \%$ for 1q21.1 duplication [17]. Two main classes of the 1q21.1 CNVs has been described. The more common Class I comprises the critical or distal region, whereas Class II compromises of the Thrombocytopenia Absent Radius (TAR) region in addition to the critical region $[15,18]$. The critical/distal region is $\approx 1.36 \mathrm{Mb}$ (from 145 to $146.35 \mathrm{Mb}$, according to NCBI build 36 ) and contain at least 12 protein coding genes, including PRKAB2, CHD1L, BCL9, ACP6, GJA5, GJA8 and NOTCH2NL [9]. Phenotypes associated with distal 1q21.1 deletion include developmental delay, cognitive impairment, microcephaly, facial anomalies, schizophrenia, attention deficit hyperactivity disorder, emotional and behavioural problems. Whereas 1q21.1 distal duplication has been associated with macrocephaly, developmental delay, autism spectrum disorder, cognitive impairment, hypertelorism and congenital cardiac anomalies $[14,15,19-21]$. Therefore, variation at this locus represents a clear risk factor for a range of neuropsychiatric disorders and need to be functionally characterised to understand the contribution of this loci to neurodevelopmental deficits leading to associated developmental psychiatric disorders. So far, the contribution of concomitantly deleted or duplicated genes in this locus towards the pathogenies of neuropsychiatric disorders is largely unknown.

To understand the impact of the Class I 1q21.1 CNV (from here referred to as 1q21.1 deletions or duplications) on neuronal development, we established a cellular model of by deriving human induced pluripotent stem cells (iPSCs) from subjects carrying 1q21.1 deletion or duplication and differentiated them into cortical neurons. We demonstrate that neural progenitor cells (NPCs) carrying 1q21.1 deletion or duplication are associated with early neurodevelopmental phenotypes. Furthermore, these NPCs after differentiation into neurons show dysregulated neuronal development, associated with altered morphology and synaptic density in comparison to controls. Moreover, these neurons are associated with dysregulated cortical layer identity. We validated aspects of these cellular phenotypes in a 1q21.1 microdeletion mouse model and show that some of these differences are conserved across species. Furthermore, we demonstrate that the presence of 1q21.1 CNVs impact the physiological and electrical properties of neurons as measured by calcium activity and multi-electrode arrays (MEAs). Finally, using iPSC derived neurons with 1q21.1 CNVs as an in vitro pharmacological model, we show that the aberrant physiological activity of these cells can be modulated by targeting $\mathrm{Ca}^{2+}$ channels.

\section{Methods}

\section{iPSC generation, characterisation and maintenance}

Fibroblasts with subject carrying 1q21.1 deletion $(n=3)$ or duplication $(n=2)$ were reprogrammed into induced pluripotent stem cells (iPSCs) using the CytoTune ${ }^{\text {TM}}$-IPS 2.0 Sendai reprogramming kit (Thermo-Fisher). Two established iPSC lines were used as controls (IBJ4 see Plumbly et al. [22] and HPSI1013i-wuye_2 purchased from HipSci). Pluripotency was confirmed by immunofluorescence, qPCR and trilineage differentiation (Supplementary Fig. 1-5). iPSCs were grown on Geltrex ${ }^{\mathrm{TM}}$ coated plates in Essential $8^{\mathrm{TM}}$ Flex media. The cell lines were genotyped to identify the location of 1q21.1 locus and to identify any pathogenetic CNVs. Further, the cell lines were regularly tested to check any mycoplasma contamination.

\section{Cortical neuronal differentiation}

iPSCs were differentiated into cortical neurons using a modified version of a previously described protocol [23]. Cells were maintained until $90-100 \%$ confluent at which point the media was changed to N2B27- (2/3 DMEM/F12, 1/3 Neurobasal, N2 supplement, B27 supplement without retinoic acid, penicillin, streptomycin, glutamine and $\beta$ mercaptoethanol) supplemented with $250 \mathrm{nM}$ LDN193189 (LDN) and $10 \mu \mathrm{M}$ SB431542 (SB). For the subsequent 10 days cells were maintained with both $\mathrm{SB}$ and LDN and then they were passaged onto fibronectin. Cells were maintained on fibronectin for 10 days in unsupplemented N2B27- media with $1 / 2$ media changes every other day. Cells were then plated onto laminin and poly-D-lysine coated plates and after 2 days the media was replaced with N2B27+ (2/3 DMEM/F12, 1/3 Neurobasal, N2 supplement, B27 supplement, penicillin, streptomycin, glutamine and $\beta$-mercaptoethanol) after a further 2 days media was replaced with N2B27+ supplemented with CultureOne ${ }^{\mathrm{TM}}$ supplement. After 2 days media was replaced with fresh N2B27+ supplemented with $5 \mu \mathrm{M}$ DAPT and $1 \mu \mathrm{M}$ PD0332991 (PD). Cells were maintained with DAPT and PD for 4 days. Cells were then dissociated using Accutase ${ }^{\circledR}$ Solution and were re-plated on laminin and poly-D-lysine coated plates at a density of $200,000 \mathrm{cells} / \mathrm{cm}^{2}$. Cells were maintained in unsupplemented N2B27+ for up to 20 days with $1 / 2$ media changes performed every other day. A minimum of three independent neuronal differentiation of all iPSC lines were done for the all the experiments reported. 


\section{A}
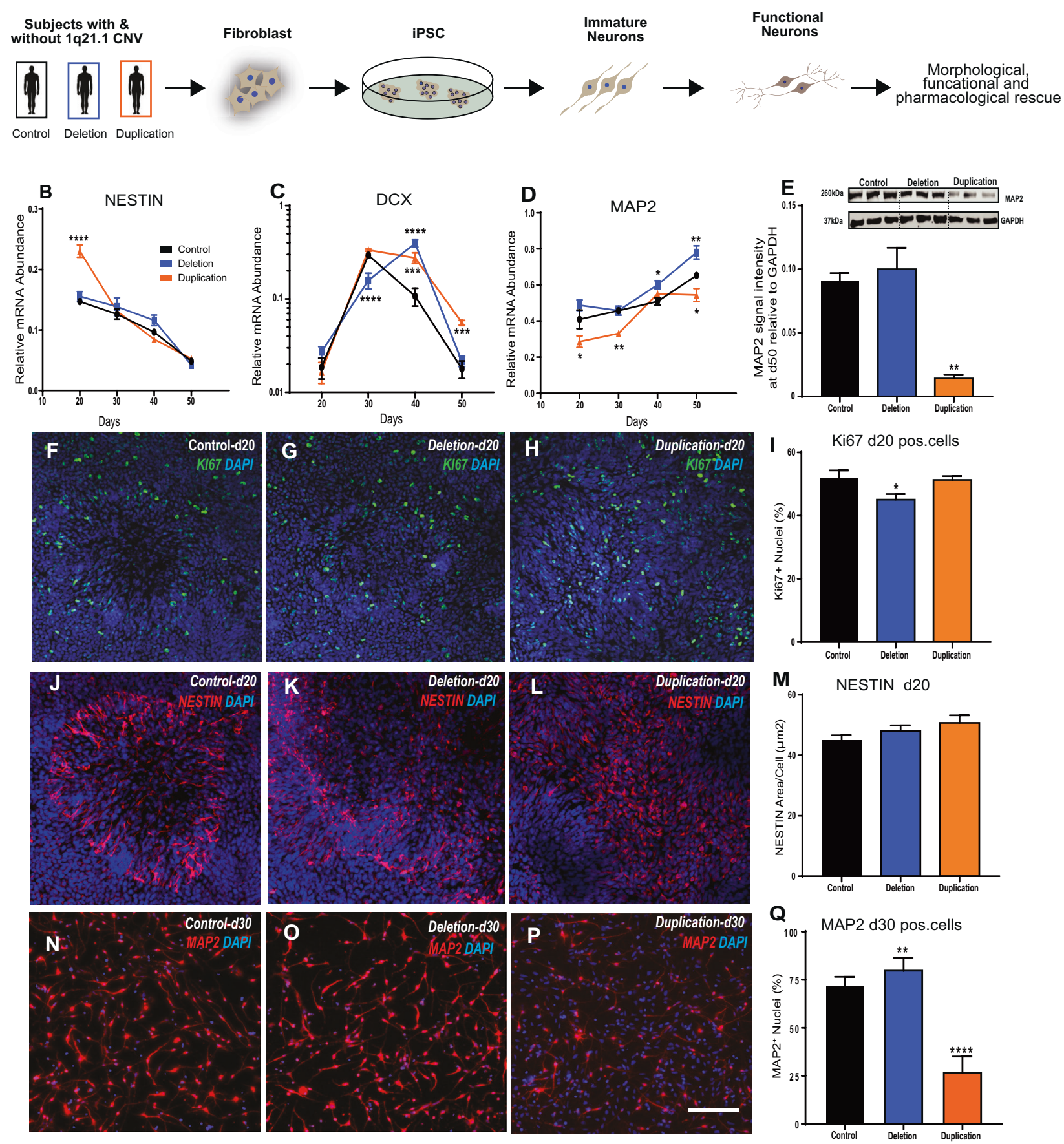

\section{Calcium imaging}

See Supplementary Methods for details

\section{Multiple electrode arrays}

See Supplementary Methods for details
Gene expression analysis, western blotting, immunofluorescence and cell counting, histological analysis of mice brains

Primers for all target genes and antibodies used are listed in Supplemental Table 2 and 3 respectively. These procedures are described in Supplementary Methods. 
Fig. 1 Cortical neurons with 1q21.1 deletion and duplications are associated with aberrant maturation. A Workflow for the generation of stable iPSC lines from human fibroblast carrying 1q21.1 deletions and duplication and the subsequent conversion of iPSC into functional neurons. B-D Gene expression analysis for the expression of NESTIN, DCX and MAP2 from day 20 to day 50 of neuronal differentiation in control, 1q21.1 deletion and 1q21.1 duplication neuronal culture. NESTIN, genotype $\left(F_{2,72}=7.94 ; P<0.001 ; n \geq 3 /\right.$ group $)$, time $\left(F_{3,72}\right.$ $=193 ; P<0.0001 ; n \geq 3 /$ group $)$; DCX, genotype $\left(F_{2,72}=7.561 ; P<\right.$ $0.0001 ; n \geq 3$ /group), time $\left(F_{3,72}=120.4 ; P<0.0001 ; n \geq 3 /\right.$ group $)$ interaction between genotype and time $\left(F_{6,72}=20.88, P<0.0001, n \geq\right.$ 3 /group); MAP2, genotype $\left(F_{2,72}=28.17 ; P<0.0001 ; n \geq 3\right.$ /group), time $\left(F_{3,72}=53.13 ; P<0.0001 ; n \geq 3\right.$ /group $)$. Data sets were analysed by two-way ANOVA with post hoc comparisons using Dunnett's multiple comparisons test comparing to control samples. Stars above points represent significance following Dunnett-corrected post hoc tests. E Representative western blot protein bands and quantitative analysis for MAP2 expression normalised to GAPDH $(n \geq 3)$. F-H Example images of KI67+ staining at day 20 of neuronal differentiation in control, 1q21.1 deletion and 1q21.1 duplication cell lines. I Quantification of the percentage of DAPI+ nuclei which colocalized with KI67 positivity in day 20 neuronal cultures $(n \geq 3)$. J-L Representative images of NESTIN+ cells at day 20 of neuronal differentiation in control, 1q21.1 deletion and 1q21.1 duplication cell lines. M Quantification of the area which staining positive for NESTIN normalised to the number of cell present in the field $(n \geq 3)$. $\mathbf{N}-\mathbf{P}$ Representative images of MAP2 positive immature neurons after 30 days of differentiation from a control, 1q21.1 deletion and 1q21.1 duplication cell line. Q Quantification of the percentage of DAPI+ nuclei that are colocalized with MAP2 positivity in immature neuronal cultures after 30 days of differentiation $(n \geq 3)$. Unless otherwise specified data were analysed using Students $T$-Tests. All data are presented as means \pm SEM $* P<0.05 ; * * P<0.01 ; * * * P<0.001$ $* * * * P<0.0001$ vs. control. Scale bar $=100 \mu \mathrm{m}$.

\section{Statistical analyses}

Data are expressed as mean \pm SEM. All data are comprised of a minimum of three separate differentiations $(n)$ for each cell line used in this study ( 2 control, 3 1q21.1 deletion and 2 1q21.1 duplication). All technical replicates were averaged before statistical testing. Statistical analyses were conducted in GraphPad Prism 6.01 (GraphPad Software). Differences between conditions or groups were evaluated using two-tailed unpaired Students $T$-Test or one/two-way ANOVA. $p$ values $<0.05$ were considered statistically significant.

\section{Results}

\section{Deletions and duplications of the 1q21.1 locus is associated with altered neuronal development}

We first assessed the effect of 1q21.1 deletion or duplication on the expression of genes within the distal 1q21.1 region, focusing on the expression of five key genes within this locus. After 50 days of differentiation three of these critical genes (BCL9, CDH1L and PRKAB2) had altered expression in 1q21.1 deletion or duplication in comparison to controls (Supplementary Fig. 6B). To determine if deletion or duplication of the 1q21.1 locus altered neurodevelopmental trajectories we quantified the expression level of: a neural stem cell marker (NESTIN [24]); a marker of immature neurons Doublecortin (DCX [25]) and a mature neuronal marker (MAP2 [26]) throughout the course of neuronal differentiation and found that the expression level in controls was in accordance with previously published studies [27, 28]. NESTIN expression was significantly higher in 1q21.1 duplication, but unchanged in the 1q21.1 deletion group after 20 days of differentiation (Fig. 1B). Similarly, other NPC markers, PAX6 and PLZF, was also elevated in duplications, but no change was seen for ZO-1 (Supplementary Fig. 7B). However, at day 20 NESTIN+ cells were similar across group (Fig. 1M). Further, we observed a small, but significant decrease of Ki67+ cells in 1q21.1 deletion culture at day 20 and a substantial decrease in Ki67 mRNA was seen at day 30 (Fig. 1F-I, Supplementary Fig. 7D). 1q21.1 duplication culture exhibit elevated Ki67 mRNA expression at day 30 (Supplementary Fig. 7D).

DCX expression in the 1q21.1 duplication group showed an increased expression at day 40 and 50. In contrast, 1q21.1 deletion cell exhibited a more complex pattern with decreased expression at day 30 , but increased expression at day 40. However, the levels were comparable to controls at day 50 (Fig. 1C). Similarly, TUJ1 expression was higher in duplication and of comparable level between deletion and controls at day 50 (Supplementary Fig. 7K-N). MAP2 expression both at protein and mRNA level were significantly reduced throughout neuronal differentiation in 1q21.1 duplication. This was further accompanied by a reduced number of MAP2 + cells at day 30 (Fig. 1D, E, Q). The 1q21.1 deletion group demonstrated an increased MAP2 expression and an increase in MAP2 + cells (Fig. 1D, Q). Although we note an increase in MAP2 protein levels at day 50, it did not reach significance (Fig. 1E).

Neuronal cell morphologies were examined at day 30 of neuronal differentiation when neuronal morphology first emerge [23] (Supplementary Fig. 8). Neurons with 1q21.1 deletion had smaller soma, whereas 1q21.1 duplication had an increased soma size. These results indicate that $1 \mathrm{q} 21.1$ $\mathrm{CNV}$ alter neuronal differentiation, and differences begin to emerge at the Neural Progenitor Cell (NPC) stage. These results show that a complex set of gene expression and protein changes occur during neurodevelopment of 1q21.1 CNV patient cells, which are unlikely to arise from a simple acceleration or retardation of the neuronal differentiation programme. However, they do suggest that the 1q21.1 duplication may delay the transition from NPC to neurons, whereas a 1q21.1 deletion suppresses proliferation and promotes neuronal production. 

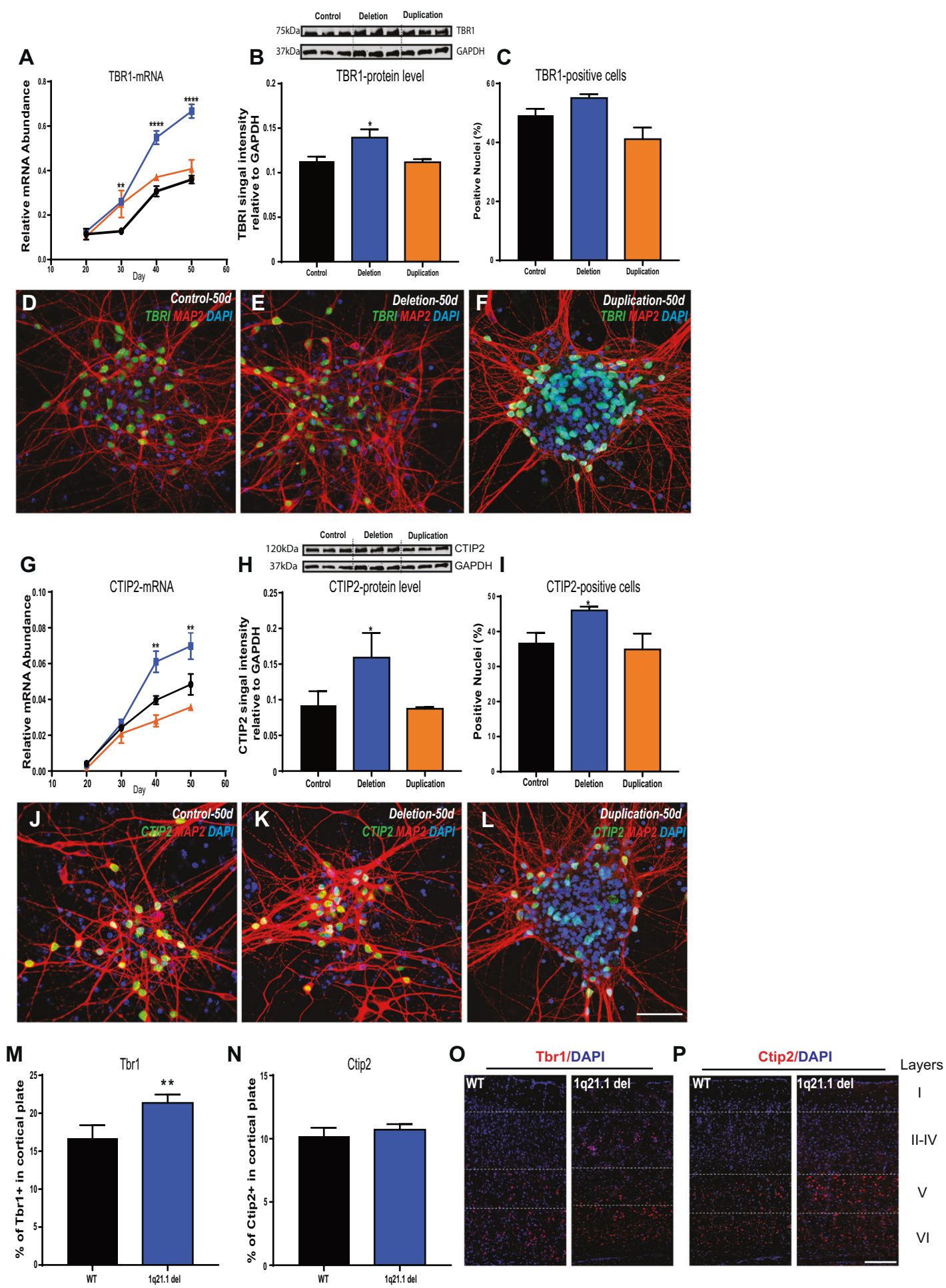

\section{Neurons with 1q21.1CNVs exhibit alterations in the cortical neuronal identity}

Alternation in corticogenesis has been linked to many developmental psychiatric disorders [29], risk for which has been associated with CNVs at 1q21.1 locus.
We therefore looked at the formation of the early born deep layer neurons specifically examining the expression of CTIP2 and TBR1. The neurons carrying 1q21.1 deletion were associated with an increased expression of TBR1 and CTIP2 both at transcript and protein level following 50 days of differentiation. These 
Fig. 2 Deletion or duplication of the 1q21.1 locus is associated with aberrant cortical neuron differentiation similar to deficits found in the 1q21.1 microdeletion mouse model. A Gene expression of cortical deep layer marker TBR1 through the course of the neuronal differentiations (from day 20 to day 50$)$. Both genotype $\left(F_{2,84}=57.55\right.$; $P<0.0001 ; n \geq 3$ /group $)$ and time $\left(F_{3,84}=365.8 ; P<0.0001 ; n \geq 3\right.$ / group) had significant effects on TBR1 expression. B Representative western blot protein bands and quantitative analysis of TBR1 expression normalised to GAPDH $(n \geq 3)$. C Quantification of the percentage of MAP2 positive cells which co-localised with TBR1 $(n \geq$ 3). D-F Representative images of MAP2 and TBR1 colocalization from a control, deletion and duplication cell line. $\mathbf{G}$ The expression of CTIP2 through the course of neuronal differentiation (from day 20 to day 50$)$. Both genotype $\left(F_{2,84}=199.7 ; P<0.0001 ; n \geq 3\right.$ /group $)$ and time $\left(F_{3,84}=133.2 ; P<0.0001 ; n \geq 3 /\right.$ group $)$ had significant effects on CTIP2 expression. H Representative western blot protein bands and quantitative analysis for CTIP2 expression normalised to GAPDH $(n \geq$ 3). I Quantification of the percentage of MAP2 positive cells which coexpress CTIP2 $(n \geq 3)$. J-L Representative images MAP2 and CTIP2 colocalization from a control, deletion and duplication cell line. Scale bar $=100 \mu \mathrm{m}$. Data sets are expressed as mean of at least three independent experiments and were analysed by Students $T$-Test or twoway ANOVA with post hoc comparisons using Dunnett's multiple comparisons test comparing to control samples. Where appropriate stars above points represent Dunnett-corrected post hoc tests. M Quantification of TBR1+ nuclei in the somatosensory cortex of adult mice modelling 1q21.1 deletion and wild type liter matched controls (WT) given as a percentage of nuclei in a $300 \mu \mathrm{m}$ section of cortex. N Quantification of CTIP2+ nuclei in the somatosensory cortex of adult mice modelling 1q21.1 microdeletion and liter matched controls given as a percentage of nuclei in a $300 \mu \mathrm{m}$ section of cortex. Data were analysed using Student's $T$-tests with six animals per group and $n \geq 3$ for each animal. All data are presented as means \pm SEM $* P<$ $0.05 ; \quad * * P<0.01 ; \quad * * * P<0.001 \quad * * * * P<0.0001$ vs. control. O Representative images of a coronal brain section of wild type (WT) and 1q21.1 microdeletion model (1q21.1) showing the expression of Tbr $1+$ cells in the somatosensory cortex of 1-month old adult mice. P Representative images of a coronal brain section of wild type (WT) and 1q21.1 microdeletion model (1q21.1) showing the expression of Ctip2+ cells in the somatosensory cortex of 1-month old adult mice. Scale $\mathrm{Bar}=100 \mu \mathrm{m}$.

results were confirmed using immunocytochemistry and indicate an increase in the number of CTIP $2+$ cells in 1q21.1 deletion. Conversely, 1q21.1 duplication was associated with a transient increase of TBR 1 expression at day 30 of differentiation and no significant change in CTIP2 expression. Furthermore, at the protein and cellular level, the expression of TBR1 and CTIP2 in 1q21.1 duplication cultures were comparable to controls (Fig. 2A-C, G-I).

To examine the potential effects that the changes seen in differentiating cells may have on brain organisation, we analysed 1-month old brains of a mouse model with a 1q21.1 microdeletion [30]. This analysis demonstrated that there was a significantly higher proportion of TBR1 cells in brains of the 1q21.1 mouse model in comparison to control littermates (Fig. 2M). These data suggest that 1q21.1 deletion result in altered cortical patterning due to an increase in the production of lower layer cortical neurons.

\section{Human neurons with 1q21.1 deletion or duplication are associated with defects in synaptogenesis}

Considering the altered differentiation potential associated with 1q21.1 deletion/duplication, we investigated the impact of the 1q21.1 CNV on synaptogenesis in our patient iPSC-derived neurons. The postsynaptic marker, PSD-95 showed a reciprocal pattern for both gene expression and protein analysis with an increased expression in 1q21.1 deletion and a decrease in 1q21.1 duplication neuronal cell (Fig. 3A, B; Supplementary Fig. 9). The presynaptic marker (synaptophysin; SYN) showed an increased gene expression and number of SYN+ puncta in the 1q21.1 deletion. On the other hand, duplication of the 1q21.1 locus was associated with a decrease of SYN+ puncta and a decrease of SYN protein level (Fig. 3C) when normalised to MAP2 (eliminating differences in morphology) (Fig. 3C-E). These results demonstrate that both 1q21.1 deletion and duplication are associated with defects in synapse development. It has previously shown that the presence of astrocytes influences the synapse formation in iPSC derived neurons [31]. Hence, we quantified the level of GFAP and S100 $\beta$ expression at day 40 and day 50, we found that level of GFAP and S100 $\beta$ was significantly minimal to the MAP2 expression (Supplementary Fig. 7I, J) and across groups.

\section{Spontaneous calcium activity reveals physiological deficits in neurons associated with 1q.21 deletion or duplications}

To begin to understand the effects of $1 \mathrm{q} 21.1 \mathrm{CNV}$ on the physiology of neurons we assessed the cytosolic dynamics of calcium using a calcium-sensitive dye. A similar proportion of cells showed spontaneous calcium activity in the control and 1q21.1 deletion cultures (Fig. 4A). However, there were significantly fewer active neuronal cells in 1q21.1 duplication cultures compared to the controls (Fig. 4A). Quantifying the rate of spontaneous calcium activity showed a significant increase in the rate of calcium events in neurons with 1q21.1 deletion (Fig. 4B). On the other hand, after excluding the inactive cells the rate of calcium events in 1q21.1 duplication cultures was similar to the controls (Fig. 4B). Finally, the amplitude of calcium signals was comparable across the groups with no significant differences between control, deletion and duplication neurons (Fig. 4C).

We then investigated the effect of the NMDA receptor antagonist AP5 (D-2-amino-5-phosphonopentanoate) and the AMPA receptor antagonist CNQX (6-Cyano-7-nitroquinoxaline-2,3-dione) in modulating the calcium signal in the neurons (Supplementary Fig. 10A). The addition of AP5 or CNQX resulted in a decrease in the percentage of active 


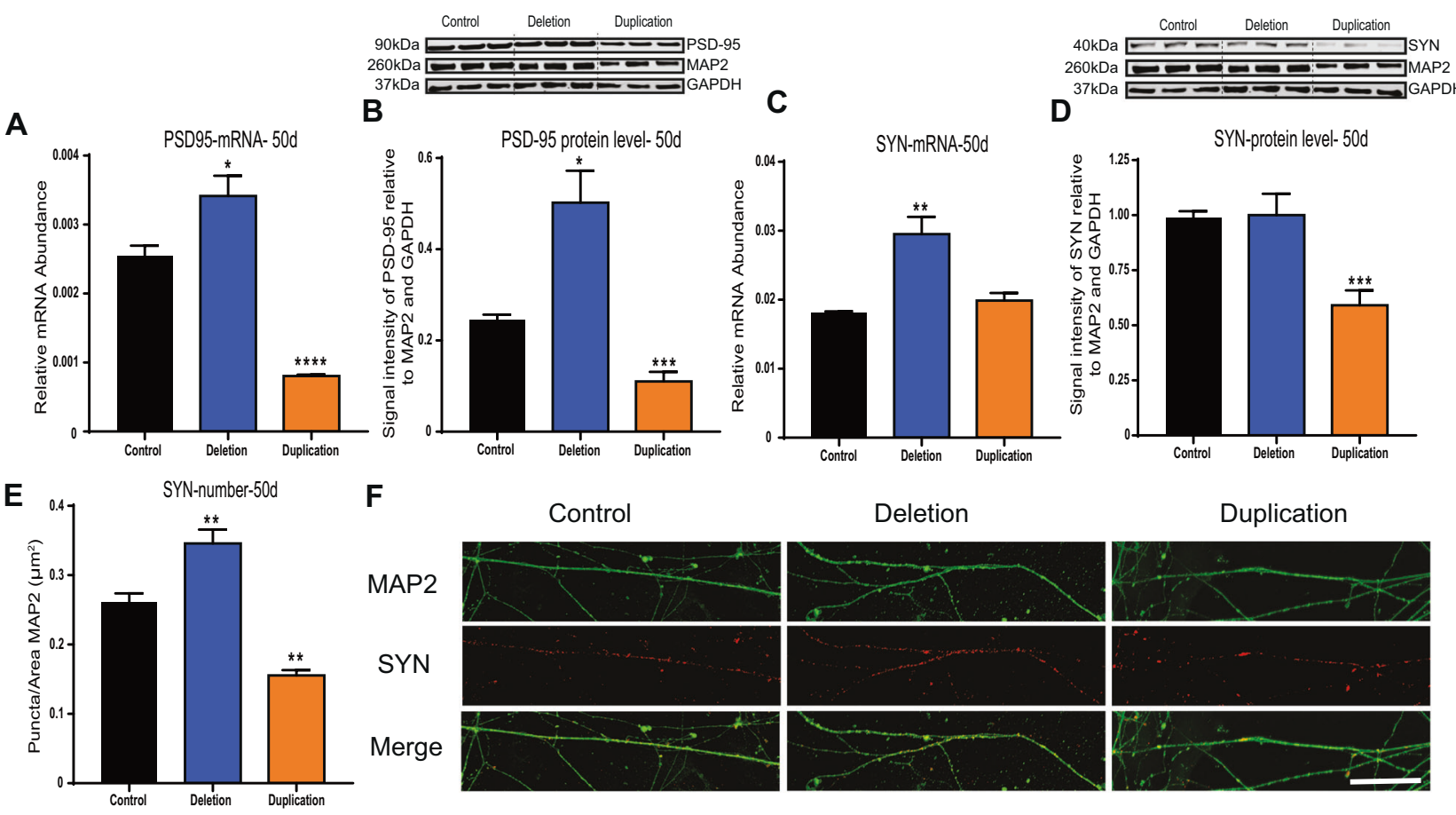

Fig. 3 Neurons with 1q21.1 deletion and duplication are associated with synaptic defects. A The expression of PSD95 mRNA at day 50 of neuronal differentiation $(n \geq 3)$. B Representative western blot protein bands and quantitative analysis for Synaptophysin expression normalised to both GAPDH and MAP2 $(n \geq 3)$. C The expression of Synaptophysin mRNA at day 50 of neuronal differentiation $(n \geq 3)$. D Representative western blot protein bands and quantitative analysis for Synaptophysin expression normalized to both GAPDH and MAP2

neurons in both the control and 1q21.1 deletion neuronal cultures. Whereas only inhibition of AMPA receptors showed a minor but significant decrease in the percentage of active neurons in 1q21.1 duplication cultures, indicating that 1q21.1 deletion neurons form connections similar to controls but the calcium activity of 1q21.1 duplication neurons could be associated with complex intricate pathways.

\section{Neurons with 1q21.1 deletion or duplication display aberrant neural network activity}

The results above indicate that 1q21.1 deletion develops rapidly, expressing neurodevelopmental genes earlier than control cells, exhibiting increased synaptogenesis and increased numbers of calcium events. In contrast, 1q21.1 duplication cells showed slow or aberrant neurodevelopment, formed fewer synapses and only $\approx 50 \%$ of neurons had active calcium signalling. We therefore examined the effect of the CNVs on neuronal network activity by use of Multi-Electrode Array (MEA) recordings [32]. Such networks are dependent on formation of functional synapses and are good indicators of neuronal deficits arising from aberrant neurodevelopment. $(n \geq 3)$. E Quantification of the number of Synaptophysin positive puncta normalised to the dendritic area stained positive for MAP2 $(n \geq$ 3). F Representative images of Synaptophysin ( $\mathrm{SYN}$, red) positive puncta in MAP2 (green) positive control, deletion and duplication neurons. Scale bar $=50 \mu \mathrm{m}$. Data were analysed using Students $T$ Tests and all data are presented as means \pm SEM $* * P<0.01$; *** $P<$ 0.001 vs. control.

Analysis of neuronal activity over a period of 50 days post plating onto the MEA showed that neurons with 1q21.1 deletion exhibited significantly higher spike rates and frequency of bursting compared to control neurons, particularly after D70 (Fig. 4F, G). In contrast, 1q21.1 duplication cells show no significant increases in either spike rate or burst rate during development. These data are consistent with the altered neuronal activity observed by calcium imaging. Later development time points on our MEA correspond to the emergence of large, synaptically connected neuronal networks, which burst fire in synchrony. 1q21.1 deletion patient cells exhibited synchronised bursting earlier in neuronal development (D70) than control cells (D100). Interestingly, the ultimate outcome for the neuronal network is not an increase in frequency of SBs between 1q21.1 deletion cultures and control cultures (Fig. 4M), but an increase SB duration (Fig. 4N), This aberrant network activity was inhibited by the NMDA inhibitor AP5 and the AMPA inhibitor NBQX (2,3-dihydroxy-6nitro-7-sulfamoyl-benzo[f]quinoxaline)

(Supplementary Fig. 10B-D), indicative of a glutamate transmitter dependent neuronal network. This is consistent with the higher synapse number seen in 1q21.1 deletion patient cells. In contrast, 1q21.1 duplication neurons show no neuronal network activity (Fig. 4J). 

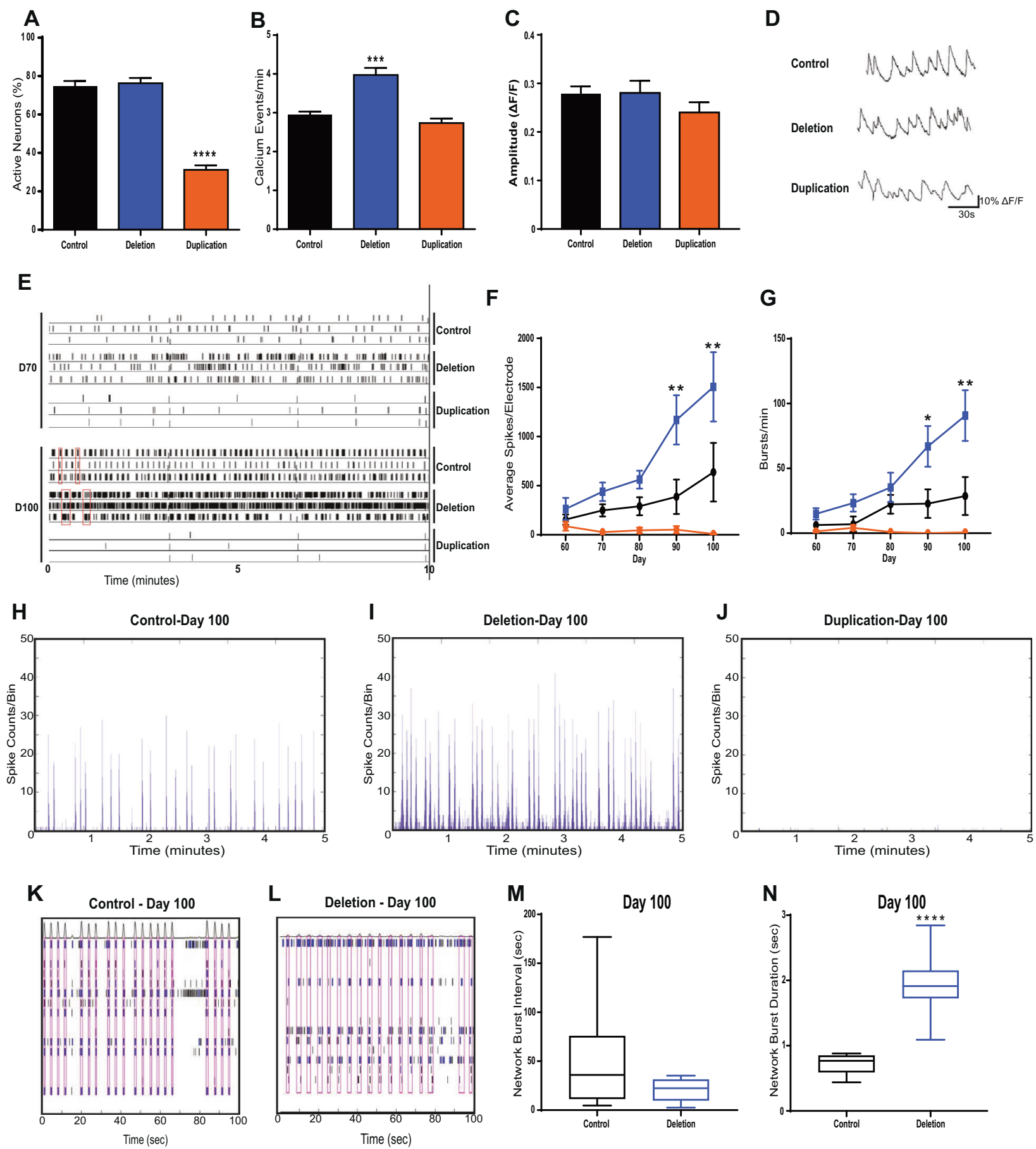

\section{Aberrant physiological activity of neurons with 1q21.1 deletion or duplications can be rescued by modulation of $\mathrm{Ca}^{2+}$ activity}

To determine a putative drug target to modulate the physiological deficits associated with 1q21.1 deletion and duplication, we first assessed the effect of 1q21.1 mutations on the expression of neuronal ion channels. Duplication of the 1q21.1 locus was associated with a decrease in the

expression of most ion channels (Fig. 5A). On the other hand, subunits of the AMPA and NMDA receptors (GLUA1 and GRIN1), and voltage-gated calcium channels CACNA1B and CACNA1E showed increased expression in 1q21.1 deletion neurons relative to control (Fig. 5A, Supplementary Fig. 11). To investigate whether a suppression of calcium signalling could reverse the increased $\mathrm{Ca}^{2+}$ spiking seen in 1q21.1 deletion neurons, we added a voltage-gated calcium channel blocker, verapamil [33, 34], 
Fig. 4 Neurons with 1q21.1 deletion and duplication display altered calcium transient activity. A Quantification of neuronal soma which show at least one characteristically neuronal calcium event $(n \geq$ 3). B Number of characteristically neuronal calcium events recorded per minute per active neurons across different groups $(n \geq 3)$. C Amplitude of calcium signals recorded from active neuronal cells across different groups. D Representative traces of calcium events as measured by changes in fluorescence from a control, deletion and duplication cell line $(n \geq 3)$. E Representative raster plot of neuronal activity exhibited by control, deletion and duplication-derived neurons at early (D70) and late (D100) neurodevelopmental stages. Red boxes indicate periods of synchronised neuronal bursts. $\mathbf{F}$ The average number of spikes recorded per electrode across the 50 days cells were maintained on MEAs. Both genotype $\left(F_{2,76}=18.06 ; P<0.0001 ; n \geq 3\right.$ / group) and time $\left(F 4_{, 76}=3.536 ; P<0.05 ; n \geq 3\right.$ /group $)$ had significant effects on the average number of spikes per electrode. $\mathbf{G}$ The average number bursts (defined as when more than three electrodes were active in the same $200 \mathrm{~ms}$ time frame) per culture across the 50 days cells were maintained on MEAs. Only genotype $\left(F_{2,61}=8.637 ; P<0.001\right.$; $n \geq 3$ /group) had a statically significant effects on the number of bursts per culture per recording. H, I, J Examples of array-wide spike detection rate (ASDR) plots, which form the basis of synchronised burst (SB) analyses. K, L Representative raster plots showing the length and interval between SBs (indicated by the red boxes) of control networks as compared to those of 1q21.1 deletion. M Quantification of network bursting interval in control and 1q21.1 deletion neurons $(n \geq$ 3). N Quantification of network burst duration in control and 1q21.1 deletion neurons $(n \geq 3)$. Data sets were analysed by Students $T$-Test or two-way ANOVA with post hoc comparisons using Dunnett's multiple comparisons test comparing to control samples. Stars above points represent Dunnett-corrected post hoc tests. All data are presented as means \pm SEM $* P<0.05 ; * * P<0.01 ; * * * P<0.001 * * * * P<0.0001$ vs. control.

to neuronal cultures. Verapamil caused a significant reduction in the rate of $\mathrm{Ca}^{2+}$ events in 1q21.1 deletion neurons (Fig. 5C). However, there was an increased rate of $\mathrm{Ca}^{2+}$ events in control neurons. These results suggest that the blockage of calcium channels can dampen the increased rate of calcium events in 1q21.1 deletion neurons.

To induce $\mathrm{Ca}^{2+}$ activity in the population of inactive cells in 1q21.1 duplication cultures, they were treated with roscovitine, which has shown to prolong the deactivation time of neuronal calcium channels [35, 36]. Addition of roscovitine significantly increased the number of calcium events in both control and 1q21.1 duplication neuronal culture (Fig. 5F). However, addition of roscovitine did not increase the proportion of spontaneously active cells in 1q21.1 duplication cultures (Fig. 5E). These results suggest that the higher and lower $\mathrm{Ca}^{2+}$ activity in 1q21.1 deletions and duplications can be modulated by targeting $\mathrm{L}$ type calcium channel antagonist and agonist.

\section{Discussion}

One of the key findings of the study is a mirrored phenotype with respect to neuronal differentiation. Deletion of the 1q21.1 locus was associated with accelerated neuronal differentiation whereas duplication of the 1q21.1 locus had negative effects on differentiation potential. These opposing phenotypes represent a possible explanation for the micro and macrocephaly associated with $\mathrm{CNVs}$ at the 1q21.1 locus. In 1q21.1 deletion subjects the accelerated differentiation may result in premature loss of proliferative precursors or in premature death of new-born neurons. In 1q21.1 duplication subjects the retention of proliferative progenitors and resistance to produce mature neurons is likely result in an increase in overall cell number. However, additional work is needed in additional cellular models together with mice model to validate these explanations.

The distal 1q21.1 region consist of at least 12 protein coding genes with the recent additions of NOTCH2NLA, NOTCH2NLB and NOTCH2NLC being of particular interest [37]. A recent study investigated the effect of NOTCH2NLB on brain development demonstrated that deletion of this gene leads to premature neuronal maturation, whereas ectopic expression lead to a delay in the differentiation of radial glial cells [38]. These results are consistent with the cellular phenotypes presented in this study and provide some evidence for the underlying mechanisms involved. Given the neurodevelopment phenotype that is associated 1q21.1 CNV in our cultures it is possible that these can be attributed to dosage variation of the NOTCH2NL gene. However, the contribution of other genes within the distal 1q21.1 locus has yet to be explored; more genetic manipulation studies are needed to elucidate the contribution of each gene towards the pathology associated with 1q21.1 CNVs and it is likely that dosage level of the genes are associated stages of neuronal differentiation.

Importantly, at a functional level deletion of the 1q21.1 distal locus was associated with increase neuronal activity and deficits in neuronal network functionality (specifically in the duration of synchronised bursts). On the other hand, duplication of the 1q21.1 locus was associated with decreased neuronal activity and an inability to form neuronal networks. These phenotypes are in part likely a result of the altered synapse production associated with CNVs at the 1q21.1 locus. However, the cause of this synaptic disparity is not clear. Our result demonstrate that altered synaptogenesis is mediated by altered expression of synapasin and PSD95. Future studies looking into expression analysis of these neurons will help in identifying other associated factors and in elucidating underlying common pathways (altered transcription/altered mRNA degradation and translation), associated with synaptogenesis. Several cellular studies looking at cellular phenotypes of other CNVs such as 2p16.3/NRXN1, 15q13.3, 16p11.2, 22q11.21 have also shown synaptic dysfunction [39-41]. Therefore, cellular dysfunction associated with CNVs (linked to psychiatric disorders) is likely to converge on deficiencies in synaptic machinery. 
A

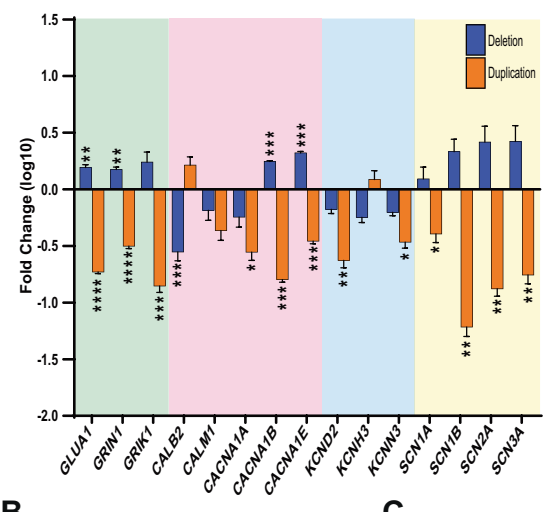

B

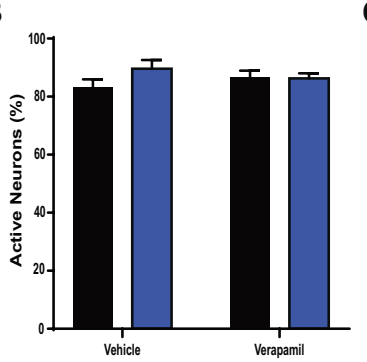

C

E

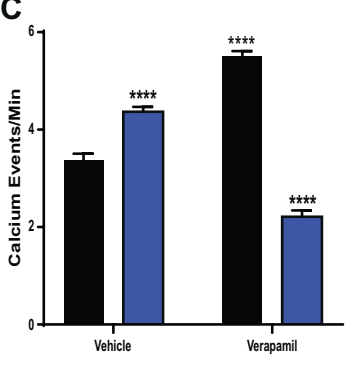

D

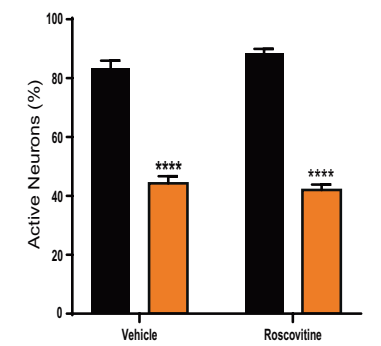

$\mathbf{F}$
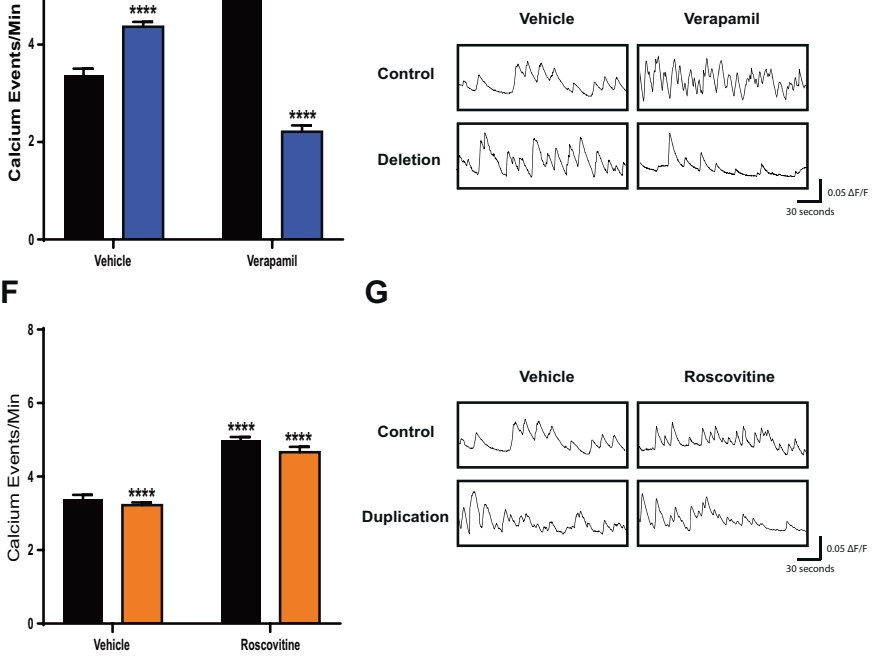

G
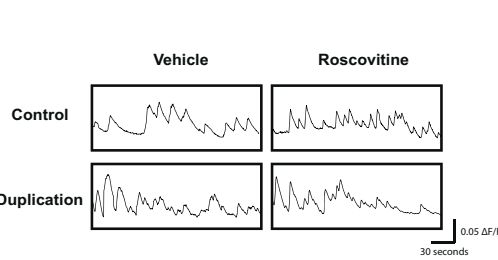

Fig. 5 Pharmacological modulation of L-type calcium channel rescues abnormal calcium activity in neurons with 1q21.1 deletions and duplications. A Expression of key neuronal channels in 1q21.1 deletion and duplication neurons following 50 days of neuronal differentiation. The values are presented as fold change compared to expression in controls. Data were analysed using multiple $T$ tests $(n \geq$ 3 ) and significance is based on Holm-Sidak corrected $P$ values. All data are presented as means \pm SEM $* P<0.05$; $* * P<0.01$; *** $P<$ $0.001 * * * * P<0.0001$ vs. control. B Quantification of neuronal soma which show at least one characteristically neuronal calcium event in day 50 control and 1q21.1 deletion culture treated for 10 days with vehicle (DMSO) or verapamil ( $n \geq 3$ /group). C Number of neuronal calcium events recorded per minute in day 50 control and 1q21.1 deletion cultures treated for 10 days with vehicle (DMSO) or verapamil. Both genotype $\left(F_{1,28}=71.64 ; P<0.0001 ; n \geq 3 /\right.$ group $)$ and the addition of verapamil $\left(F_{2,28}=79.56 ; P<0.0001 ; n \geq 3\right.$ /group $)$ had significant effects on the average rate of calcium events. Furthermore, there was a significant interaction between the effect of genotype and drug $\left(F_{2,28}=162.4 ; P<0.0001 ; n \geq 3 /\right.$ group $)$ on the rate of calcium events. D Example traces of single neurons from both control and

Our results demonstrate that addition of verapamil (a calcium channel antagonist) could reduce the rate of calcium transients in 1q21.1 deletion neurons. While some studies have questioned the specificity of verapamil $[42,43]$, our results suggests that the changes in calcium dynamics seen in 1q21.1 deletion neurons can be modulated

1q21.1 deletion neurons treated with vehicle or verapamil. E Quantification of soma which show at least one characteristically neuronal calcium event in day 50 control and 1q21.1 deletion cultures treated for 10 days with vehicle (DMSO) or roscovitine. Only genotype had a significant effect on the percentage of active cells $\left(F_{1,22}=463.9 ; P<\right.$ $0.0001 ; n \geq 3$ /group). $\mathbf{F}$ Number of characteristically neuronal calcium events recorded per minute in day 50 control and 1q21.1 duplication cultures treated for 10 days with vehicle (DMSO) or roscovitine. Both genotype $\left(F_{1,22}=38.1 ; P<0.0001 ; n \geq 3\right.$ /group $)$ and the addition of roscovitine $\left(F_{2,22}=63.87 ; P<0.0001 ; n \geq 3\right.$ /group $)$ had significant effects on the average rate of calcium events. Furthermore, there was a significant interaction between the effect of genotype and drug $\left(F_{2,22}=16.06 ; P<0.0001 ; n \geq 3\right.$ /group $)$ on the rate of calcium events. G Representative traces of single neurons from both control and 1q21.1 duplication cultures treated with vehicle or roscovitine. Data sets were analysed by two-way ANOVA with post hoc comparisons using Dunnett's multiple comparisons test comparing to control vehicle treated samples. Stars above points represent Dunnettcorrected post hoc tests. All data are presented as means \pm SEM; $* * * P<0.001 * * * * P<0.0001$ vs. vehicle treated control.

by altering the activity of voltage-gated calcium channels. The contribution and involvement of other calcium channels however cannot be ruled out. While verapamil has been used as a treatment for bipolar disorder, albeit with limited sucess [44], it has been shown to improve scopolamineinduced memory impairments in mice $[45,46]$. 
Roscovitine was used in an attempt to induce calcium activity in inactive 1q21.1 duplication cells by inhibiting cell cycle progression and modulating calcium channel activity $[35,47]$. The addition of roscovitine was able to increase calcium activity in 1q21.1 duplication neurons consistent with previous studies [48]. However, roscovitine failed to increase the proportion of active neurons in either the control of 1q21.1 duplication group.

The present study focussed largely on identifying broad classes of neuronal dysfunction and therefore further work is necessary to elucidate the precise molecular mechanisms which underly the cellular phenotypes identified in this study. Critically future work using global transcriptomic analysis may help in identifying the precise genetic mechanisms underlying the dysfunctions identified in this study.

Acknowledgements We thank Craig Joyce and Olena Peter for their help in reprogramming cells and Dr. Faraz Mahmood Ali of the Dept. of Dermatology, University Hospital Wales for the skin biopsies.

Funding This work was supported by CMU fellowship to YAS. GC, AJH and $\mathrm{JH}$ are supported by TWF Changing Minds Programme. JH, AJH, DEJL and MO is supported by Welcome (DEFINE Strategic Award 100202/Z/12/Z). JH is also supported by Hodge Foundation (Centre Grant) and MRC grants (MR/ L010305/1, G0800509, MR/ NO22572/1 and MR/L011166/1). MBMvdB is supported by MRC grants (MR/N022572/1 and MR/L011166/1). The work was also support from core facilities of the Neuroscience and Mental Health Research Institute, Cardiff University, UK and the National Centre for Mental Health was supported with funds from Health and Care Research Wales.

Author contributions Conceptualisation: YAS; Planning, design and instigation: GC, YAS; Investigation, analysis and methodology: GC, MA, SL, TS, YAS; Resources including participant testing: JH, DEJL, MvdB, MO, MZ; Figures and initial writing; GC, YAS; Writing and editing of manuscript; GC, JH, AJH, YAS. All authors have read and reviewed the manuscript.

\section{Compliance with ethical standards}

Conflict of interest The authors declare no competing interests.

Ethics statement Generation and use of human iPSC were approved by the Cardiff University and HSE (GMO130/19.3). Clinical and psychometric testing (supplementary table 1) of participants and skin biopsies was approved by the Regional Ethics Committee of the National Health Service (study 14/WA/0035).

Publisher's note Springer Nature remains neutral with regard to jurisdictional claims in published maps and institutional affiliations.

Open Access This article is licensed under a Creative Commons Attribution 4.0 International License, which permits use, sharing, adaptation, distribution and reproduction in any medium or format, as long as you give appropriate credit to the original author(s) and the source, provide a link to the Creative Commons license, and indicate if changes were made. The images or other third party material in this article are included in the article's Creative Commons license, unless indicated otherwise in a credit line to the material. If material is not included in the article's Creative Commons license and your intended use is not permitted by statutory regulation or exceeds the permitted use, you will need to obtain permission directly from the copyright holder. To view a copy of this license, visit http://creativecommons. org/licenses/by/4.0/.

\section{References}

1. Cooper GM, Coe BP, Girirajan S, Rosenfeld JA, Vu TH, Baker C, et al. A copy number variation morbidity map of developmental delay. Nat Genet. 2011;43:838-46.

2. Gilissen C, Hehir-Kwa JY, Thung DT, van de Vorst M, van Bon $\mathrm{BW}$, Willemsen $\mathrm{MH}$, et al. Genome sequencing identifies major causes of severe intellectual disability. Nature. 2014;511:344-7.

3. Krumm N, Turner TN, Baker C, Vives L, Mohajeri K, Witherspoon $\mathrm{K}$, et al. Excess of rare, inherited truncating mutations in autism. Nat Genet. 2015;47:582-8.

4. Marshall CR, Howrigan DP, Merico D, Thiruvahindrapuram B, $\mathrm{Wu} \mathrm{W}$, Greer DS, et al. Contribution of copy number variants to schizophrenia from a genome-wide study of 41,321 subjects. Nat Genet. 2017;49:27-35.

5. Williams NM, Zaharieva I, Martin A, Langley K, Mantripragada $\mathrm{K}$, Fossdal R, et al. Rare chromosomal deletions and duplications in attention-deficit hyperactivity disorder: a genome-wide analysis. Lancet. 2010;376:1401-8.

6. Chawner S, Owen MJ, Holmans P, Raymond FL, Skuse D, Hall J, et al. Genotype-phenotype associations in children with copy number variants associated with high neuropsychiatric risk in the UK (IMAGINE-ID): a case-control cohort study. Lancet Psychiatry. 2019;6:493-505.

7. Eaton CB, Thomas RH, Hamandi K, Payne GC, Kerr MP, Linden $\mathrm{DEJ}$, et al. Epilepsy and seizures in young people with $22 \mathrm{q} 11.2$ deletion syndrome: Prevalence and links with other neurodevelopmental disorders. Epilepsia. 2019;60:818-29.

8. Kirov G. CNVs in neuropsychiatric disorders. Hum Mol Genet. 2015;24:R45-49.

9. Torres F, Barbosa M, Maciel P. Recurrent copy number variations as risk factors for neurodevelopmental disorders: critical overview and analysis of clinical implications. J Med Genet. 2016;53:73-90.

10. Niarchou M, Chawner S, Doherty JL, Maillard AM, Jacquemont S, Chung WK, et al. Psychiatric disorders in children with 16p11.2 deletion and duplication. Transl Psychiatry. 2019;9:8.

11. Woodbury-Smith M, Paterson AD, Thiruvahindrapduram B, Lionel AC, Marshall CR, Merico D, et al. Using extended pedigrees to identify novel autism spectrum disorder (ASD) candidate genes. Hum Genet. 2015;134:191-201.

12. Neale BM, Kou Y, Liu L, Ma'ayan A, Samocha KE, Sabo A, et al. Patterns and rates of exonic de novo mutations in autism spectrum disorders. Nature. 2012;485:242-5.

13. Ruderfer DM, Hamamsy T, Lek M, Karczewski KJ, Kavanagh D, Samocha KE, et al. Patterns of genic intolerance of rare copy number variation in 59,898 human exomes. Nat Genet. 2016;48:1107-11.

14. Mefford HC, Sharp AJ, Baker C, Itsara A, Jiang Z, Buysse K, et al. Recurrent rearrangements of chromosome 1q21.1 and variable pediatric phenotypes. N. Engl J Med. 2008;359:1685-99.

15. Brunetti-Pierri N, Berg JS, Scaglia F, Belmont J, Bacino CA, Sahoo T, et al. Recurrent reciprocal 1q21.1 deletions and duplications associated with microcephaly or macrocephaly and developmental and behavioral abnormalities. Nat Genet. 2008;40:1466-71.

16. Sharp AJ, Locke DP, McGrath SD, Cheng Z, Bailey JA, Vallente $\mathrm{RU}$, et al. Segmental duplications and copy-number variation in the human genome. Am J Hum Genet. 2005;77:78-88. 
17. Owen D, Bracher-Smith M, Kendall KM, Rees E, Einon M, Escott-Price V, et al. Effects of pathogenic CNVs on physical traits in participants of the UK Biobank. BMC Genomics. 2018;19:867.

18. Dolcetti A, Silversides CK, Marshall CR, Lionel AC, Stavropoulos DJ, Scherer SW, et al. 1q21.1 Microduplication expression in adults. Genet Med. 2013;15:282-9.

19. Buse M, Cuttaia HC, Palazzo D, Mazara MV, Lauricella SA, Malacarne M, et al. Expanding the phenotype of reciprocal 1q21.1 deletions and duplications: a case series. Ital $\mathrm{J}$ Pediatr. 2017;43:61.

20. Rosenfeld JA, Traylor RN, Schaefer GB, McPherson EW, Ballif BC, Klopocki E, et al. Proximal microdeletions and microduplications of 1q21.1 contribute to variable abnormal phenotypes. Eur J Hum Genet. 2012;20:754-61.

21. Cunningham AC, Hall J, Einfeld S, Owen MJ, consortium I-I, van den Bree MBM. Assessment of emotions and behaviour by the Developmental Behaviour Checklist in young people with neurodevelopmental CNVs. Psychol Med. 2020:1-13. https://www.ca mbridge.org/core/journals/psychological-medicine/article/a ssessment-of-emotions-and-behaviour-by-the-developmental-beha viourchecklist-in-young-people-with-neurodevelopmental-cnvs/ D26B0837601A9C195D3534A86670EA02.

22. Plumbly W, Brandon N, Deeb TZ, Hall J, Harwood AJ. L-type voltage-gated calcium channel regulation of in vitro human cortical neuronal networks. Sci Rep. 2019;9:13810.

23. Chambers SM, Qi Y, Mica Y, Lee G, Zhang XJ, Niu L, et al. Combined small-molecule inhibition accelerates developmental timing and converts human pluripotent stem cells into nociceptors. Nat Biotechnol. 2012;30:715-20.

24. Lendahl U, Zimmerman LB, McKay RD. CNS stem cells express a new class of intermediate filament protein. Cell. 1990;60:585-95.

25. Gleeson JG, Lin PT, Flanagan LA, Walsh CA. Doublecortin is a microtubule-associated protein and is expressed widely by migrating neurons. Neuron. 1999;23:257-71.

26. Harada A, Teng J, Takei Y, Oguchi K, Hirokawa N. MAP2 is required for dendrite elongation, PKA anchoring in dendrites, and proper PKA signal transduction. J Cell Biol. 2002;158:541-9.

27. Pasca SP, Portmann T, Voineagu I, Yazawa M, Shcheglovitov A, Pasca AM, et al. Using iPSC-derived neurons to uncover cellular phenotypes associated with Timothy syndrome. Nat Med. 2011;17:1657-62.

28. Meyer K, Feldman HM, Lu T, Drake D, Lim ET, Ling KH, et al. REST and neural gene network dysregulation in iPSC models of Alzheimer's disease. Cell Rep. 2019;26:1112-27. e1119

29. Rubenstein JL. Annual research review: development of the cerebral cortex: implications for neurodevelopmental disorders. J Child Psychol Psychiatry. 2011;52:339-55.

30. Nielsen J, Fejgin K, Sotty F, Nielsen V, Mork A, Christoffersen $\mathrm{CT}$, et al. A mouse model of the schizophrenia-associated 1q21.1 microdeletion syndrome exhibits altered mesolimbic dopamine transmission. Transl Psychiatry. 2017;7:1261.

31. Klapper SD, Garg P, Dagar S, Lenk K, Gottmann K, Nieweg K. Astrocyte lineage cells are essential for functional neuronal differentiation and synapse maturation in human iPSC-derived neural networks. Glia. 2019;67:1893-909.

32. Odawara A, Katoh H, Matsuda N, Suzuki I. Physiological maturation and drug responses of human induced pluripotent stem cell-derived cortical neuronal networks in long-term culture. Sci Rep. 2016;6:26181.
33. Dobrev D, Milde AS, Andreas K, Ravens U. The effects of verapamil and diltiazem on N-, P- and Q-type calcium channels mediating dopamine release in rat striatum. $\mathrm{Br} \mathrm{J}$ Pharm. 1999;127:576-82.

34. MacDonald JF, Schneiderman JH. Actions of verapamil on the excitability of cultured neurones. Can J Physiol Pharm. 1983;61:1467-72.

35. Buraei Z, Anghelescu M, Elmslie KS. Slowed N-type calcium channel (CaV2.2) deactivation by the cyclin-dependent kinase inhibitor roscovitine. Biophys J. 2005;89:1681-91.

36. Tarr TB, Malick W, Liang M, Valdomir G, Frasso M, Lacomis D, et al. Evaluation of a novel calcium channel agonist for therapeutic potential in Lambert-Eaton myasthenic syndrome. J Neurosci. 2013;33:10559-67.

37. Steinberg KM, Schneider VA, Graves-Lindsay TA, Fulton RS, Agarwala R, Huddleston J, et al. Single haplotype assembly of the human genome from a hydatidiform mole. Genome Res. 2014;24:2066-76.

38. Fiddes IT, Lodewijk GA, Mooring M, Bosworth CM, Ewing AD, Mantalas GL, et al. Human-specific NOTCH2NL genes affect notch signaling and cortical neurogenesis. Cell. 2018;173:1356-69. e1322

39. Uddin M, Unda BK, Kwan V, Holzapfel NT, White SH, Chalil L, et al. OTUD7A regulates neurodevelopmental phenotypes in the $15 \mathrm{q} 13.3$ microdeletion syndrome. Am J Hum Genet. 2018;102:278-95.

40. Deshpande A, Yadav S, Dao DQ, Wu ZY, Hokanson KC, Cahill $\mathrm{MK}$, et al. Cellular phenotypes in human iPSC-derived neurons from a genetic model of autism spectrum disorder. Cell Rep. 2017;21:2678-87.

41. Gokhale A, Hartwig C, Freeman AAH, Bassell JL, Zlatic SA, Sapp Savas C, et al. Systems analysis of the 22q11.2 microdeletion syndrome converges on a mitochondrial interactome necessary for synapse function and behavior. J Neurosci. 2019;39:3561-81.

42. Cai D, Mulle JG, Yue DT. Inhibition of recombinant $\mathrm{Ca} 2+$ channels by benzothiazepines and phenylalkylamines: classspecific pharmacology and underlying molecular determinants. Mol Pharm. 1997;51:872-81.

43. Tarabova B, Lacinova L, Engel J. Effects of phenylalkylamines and benzothiazepines on $\mathrm{Ca}(\mathrm{v}) 1.3$-mediated $\mathrm{Ca} 2+$ currents in neonatal mouse inner hair cells. Eur J Pharm. 2007;573:39-48.

44. Cipriani A, Saunders K, Attenburrow MJ, Stefaniak J, Panchal P, Stockton S, et al. A systematic review of calcium channel antagonists in bipolar disorder and some considerations for their future development. Mol Psychiatry. 2016;21:1324-32.

45. Biala G, Kruk-Slomka M, Jozwiak K. Influence of acute or chronic calcium channel antagonists on the acquisition and consolidation of memory and nicotine-induced cognitive effects in mice. Naunyn Schmiedebergs Arch Pharm. 2013;386:651-64.

46. Woodside BL, Borroni AM, Hammonds MD, Teyler TJ. NMDA receptors and voltage-dependent calcium channels mediate different aspects of acquisition and retention of a spatial memory task. Neurobiol Learn Mem. 2004;81:105-14.

47. Yarotskyy V, Gao G, Du L, Ganapathi SB, Peterson BZ, Elmslie KS. Roscovitine binds to novel L-channel (CaV1.2) sites that separately affect activation and inactivation. J Biol Chem. 2010;285:43-53.

48. Tomizawa K, Ohta J, Matsushita M, Moriwaki A, Li ST, Takei K, et al. Cdk5/p35 regulates neurotransmitter release through phosphorylation and downregulation of P/Q-type voltage-dependent calcium channel activity. J Neurosci. 2002;22:2590-7. 